Mots. Les langages du politique

$93 \mid 2010$

Figures et filiations dans le discours politique latinoaméricain

\title{
Construction et évolution du discours du Partido dos Trabalhadores au Brésil (1980-2006). Une approche dialogique
}

Miguel Espar Argerich et Dóris de Arruda Carneiro da Cunha

\section{OpenEdition}

Journals

Édition électronique

URL : https://journals.openedition.org/mots/19833

DOI : $10.4000 /$ mots. 19833

ISSN : 1960-6001

Éditeur

ENS Éditions

Édition imprimée

Date de publication : 1 juillet 2010

Pagination : 127-143

ISBN : 978-2-84788-231-5

ISSN : 0243-6450

Référence électronique

Miguel Espar Argerich et Dóris de Arruda Carneiro da Cunha, «Construction et évolution du discours du Partido dos Trabalhadores au Brésil (1980-2006). Une approche dialogique », Mots. Les langages du politique [En ligne], 93 | 2010, mis en ligne le 01 octobre 2012, consulté le 24 avril 2022. URL : http:// journals.openedition.org/mots/19833; DOI : https://doi.org/10.4000/mots.19833 


\section{Construction et évolution du discours du Partido dos Trabalhadores au Brésil (1980-2006). Une approche dialogique}

À l'heure où, dans les pays avancés, la question du contenu idéologique des actes, des discours et des actes de discours est moins que jamais «à la mode», il nous a semblé intéressant d'appliquer les grilles de lecture issues de la théorie bakhtinienne du langage à un discours de propagande politique qui se déploie largement, au Brésil, hors des moyens de communication et des médias constitués, presse écrite, radio et télévision, lesquels sont pour la plupart acquis à l'opposition conservatrice. Fortement démocratique et antilibéral, ce discours a acquis une prise sur le réel - surtout depuis que Luiz Inácio Lula da Silva, dit Lula, leader du Parti des Travailleurs, a accédé à la présidence

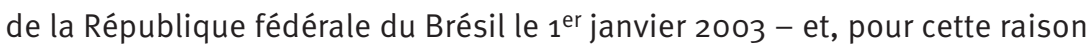
même, constitue actuellement l'enjeu d'une lutte politique tendue.

Le parcours discursif du Parti des Travailleurs nous semble particulièrement intéressant à suivre. C'est celui d'un parti de gauche qui a accédé au pouvoir dans un temps relativement court - vingt-deux ans -, à la suite d'une évolution marquée par des changements importants. De mouvement d'opinion, ce parti est devenu parti de gouvernement; fondé par des opposants à la dictature militaire mobilisés par des intellectuels, cadres, étudiants et militants ouvriers très actifs, il a accédé aux responsabilités du pouvoir et dirige l'alliance politique tactique qui gouverne aujourd'hui le pays. Corrélativement, il est passé d'un discours radical de phraséologie marxiste à un autre plus consensuel, tempéré par l'expérience et par les exigences de la pratique du pouvoir.

La problématique qui inspire cette recherche ${ }^{1}$ tient à ce que, dans les mois qui ont suivi l'arrivée de Lula à la magistrature suprême, a prévalu l'idée que

Université fédérale du Pernambouc

horespar@yahoo.com

Université fédérale du Pernambouc et CNPQ

doris@aufpe.br

1. Cette étude s'appuie largement sur la thèse de doctorat soutenue par Miguel Espar Argerich à l'Université fédérale de Pernambouc en 2007, sous la direction de Dóris Cunha, «Dialogismo em discursos do Partido dos Trabalhadores », dont nous reprenons ici quelques-unes des données et des analyses. Nous remercions Marc Arabyan pour la relecture de ce texte et de nos traductions. 
le président de la République et son parti avaient «trahi le peuple» : leur prise du pouvoir n'aurait été suivie d'aucun changement, ni dans la politique économique du pays, ni dans la façon de gouverner, le Parti des Travailleurs ayant fait alliance avec des partis du centre et de la droite parlementaire qui participent traditionnellement au gouvernement du Brésil. Dans quelle mesure le discours de ce parti a-t-il changé depuis sa création ? De quel point de vue peut-on parler de changement de discours ? Ce changement, s'il est avéré, constituet-il une trahison ou au contraire le signe d'une consolidation démocratique? Telles sont les questions à partir desquelles nous avons analysé un corpus de textes programmatiques pour suivre leurs relations interdiscursives.

Faute de place, nous ne présenterons ici que trois de ces textes, retenus parmi un très grand nombre d'autres textes accessibles en ligne², pour cette raison qu'ils constituent le point de départ et le point d'arrivée de cette évolution. D’un côté, la Carta de Princípios (désormais Carta) du $1^{\mathrm{er}}$ mai 1979, "Charte de principes» des dirigeants syndicaux de la métallurgie appelant à la fondation du Parti des Travailleurs, suivie du Manifeste du parti (désormais Manifesto), du 10 février 1980, texte fondateur de ladite organisation; de l'autre, vingt-six ans après la fondation du parti et au bout de trois ans d'exercice du pouvoir, les Deliberações ("Délibérations») de la Treizième rencontre nationale du Partido dos Trabalhadores en septembre 2006.

Observant les unités lexicales en relation avec leurs conditions d'énonciation, nous avons cherché les implications et restrictions de sens activées dans le choix des mots et leurs contextes pour identifier les voix issues de la conscience et de la mémoire sociales et politiques de la gauche brésilienne incarnée par le Parti des Travailleurs (celle-ci regroupe différents courants communistes, socialistes et syndicalistes révolutionnaires, ainsi que des communautés ecclésiales de base issues de la théologie de la libération, notamment après le concile Vatican II -1962-1965 - et la conférence épiscopale de Medellin en 1968). Pour le dire vite, nous nous sommes inspirés de la conception bakhtinienne de l'énonciation et de l'énoncé dans une perspective dialogique, suivant les fils conducteurs identifiés par l'analyse du discours comme révélateurs des intérêts constitutifs de l'évènement discursif - nous y reviendrons.

Comme on le sait, le coup d'État du 31 mars 1964 a mis un terme au gouvernement socialisant du président João Goulart dans un contexte de guerre froide marqué, en Amérique latine, par la montée de nationalismes qui revendiquent l'autonomie des pays du Tiers-Monde dans les relations internationales et la mise en place d'États interventionnistes et protectionnistes (Ferreira, 2003). Commence une longue dictature civile-militaire. La période 1969-1974 est la plus dure: on l'appelle «les années de plomb », étant donné le niveau de

2. [URL : http://www.pt.org.br/portalpt/documentos/documentos-historicos-32/pagina-1/]. Consulté le 20 avril 2010. 
régression politico-sociale et de répression policière - des centaines de morts et de disparus - qui limite les espaces de liberté et de débat à des institutions tolérées - l'Église catholique, les syndicats, les associations - ou au domicile des particuliers. À partir de 1975 commence un lent processus d'ouverture qui trouvera son aboutissement en 1985 avec le retour à la liberté politique, et en 1988 avec le vote de la nouvelle Constitution.

En 1976, à Osasco (État de São Paulo), les ouvriers de l'industrie obtiennent des augmentations de salaires en se mettant en grève, suivis, en 1978 et 1979, par ceux de l'ABC3 (Alves, 2005, p. 286-317; Santana, 2003, p. 286-299). Ces mouvements sont l'expression du mécontentement social et politique de nombreux secteurs du salariat et de la société civile, signes tangibles de révolte contre le régime militaire. Mais les résultats obtenus sont loin des espérances parce que les classes moyennes - qui voient leurs revenus s'améliorer - restent fidèles au pacte politique qui légitime la présence des militaires au pouvoir avec le soutien de la bourgeoisie d'affaires, classe d'entrepreneurs nationaux protégés par les barrières douanières que le nouveau régime a mises en place.

La prise de conscience des limites des moyens de lutte adoptés par les mouvements sociaux est à l'origine de la décision de fonder un parti politique réunissant les travailleurs pour représenter leurs intérêts, avec pour premiers protagonistes les syndicalistes de la métallurgie qui officialisent, dès 1979, leur projet de Partido dos Trabalhadores et appellent l'ensemble des travailleurs brésiliens à s'engager dans sa construction (Skidmore, 1988, p. 427-432). Ainsi un parti entièrement nouveau par sa composition et ses objectifs surgit-il dans le paysage politique brésilien, alors que la dictature commence à envisager le retour du pays à la démocratie. C'est une surprise pour le personnel politique en place, dans la mesure où le Parti des Travailleurs réussit d'emblée à coaliser, au nom d'un intérêt commun, un ensemble de forces jusqu'alors dispersées : communistes de toutes obédiences, chrétiens progressistes, démocrates convaincus, leaders des communautés rurales, cols bleus mais aussi cols blancs. Malgré le caractère hétérogène, pour ne pas dire hétéroclite, de cette coalition - ou, qui sait, grâce à son action catalysante -, le jeune parti développe sa visée démocratique - arriver au pouvoir et s'y maintenir par les urnes - comme un objectif stratégique. Après les grandes manifestations de 1983 suivies de la restauration progressive des libertés publiques, son cinquième congrès (dont nous ne pouvons rendre compte ici, faute de place) voit le Parti des Travailleurs s'engager dans les perspectives ouvertes par l'élection au suffrage universel direct du président de la République, qui sera mise en place par la Constitution de 1988. Ce congrès décide un recentrage politique en direction des classes moyennes et commence à passer des alliances.

3. ABC, sigle formé à partir de Santo André, São Bernardo et São Caetano, désigne la zone délimitée par trois des principales communes industrielles de la banlieue de São Paulo. 
Suivent par étapes son arrivée à la direction des principaux États et métropoles du Brésil, puis l'élection de Lula à la présidence en 2002, avec 52,8 millions de voix (79,53\% des votants) ${ }^{4}$, et sa réélection en 2006 avec 58,3 millions de voix (81,01\% des votants).

\section{Méthodologie}

\section{Dialogisme et discours politique}

Le dialogisme est ici compris comme un principe intrinsèque au discours 5 : les énoncés «dialoguent» entre eux, ils se reprennent en se modifiant et se répondent, tout en étant traversés par des jugements et des visées sociales et politiques multiples. Ce point de vue théorique nous a conduits à considérer chaque discours comme l'expression concrète d'un dialogue des voix dans chaque énonciation, d'où son hétérogénéité constitutive (Authier-Revuz, 1982).

De ce point de vue, le mot reproduit et reflète, de façon voilée sinon masquée, des conflits de classe, de genre, de génération, de religion, de culture, et plus généralement de groupes d'appartenance. Il est le produit de l'interaction des voix et, chargé d'idéologie, l'expression des interactions sociales. Inséparable du procès énonciatif, comprenant le verbal et le non-verbal, l'énoncé témoigne des intérêts en jeu et en lutte dans la société. Dans les premières pages de Pour une philosophie de l'acte, ouvrage posthume composé dans les années vingt, Bakhtine décrit une sémiotique dans laquelle le mot n'a pas de référent objectif : le simple fait de commencer à parler d'un objet, écrit-il en substance, signifie que j'ai pris position à son sujet - non pas une position indifférente, mais une position intéressée. Par son intonation, l'énoncé signifie mon évaluation de son objet, mon opinion sur ce qu'il y a de désirable ou d'indésirable en lui. Tout discours reflète ainsi les voix, les propos, les évènements, les situations et les intérêts des parties qui y sont impliquées.

4. Après trois échecs en 1989, 1994 et 1998. L'élément décisif de la réussite aura été l'approbation, par la Convention nationale du Parti des Travailleurs, en juin 2002, d'une large alliance politique avec les partis du centre et de gauche, sur un programme de gouvernement fondé sur le rachat de la dette extérieure (voir le site de la présidence, http://www.presidencia.gov.br / presidente/, consulté le 19 avril 2010).

5. Nous partageons le point de vue de Frédéric François sur la difficulté qu'il y a à utiliser de «grands mots » comme dialogisme, sens ou genre et à choisir ce qui serait le «bon mot» ou la «bonne expression » : hétérologie sémiotique ou plutôt, hétérologie des sens-forces ? C'est barbare. Polyphonie est au contraire presque trop simple, et fait disparaitre la question du mode d'accord ou de désaccord des voix en question (voir François, 2006, p. 84-87). 


\section{Les genres}

Une carta, un manifesto et des deliberações, autant de genres différents sur lesquels il convient de s'expliquer pour commencer. La place manque ici pour présenter ne serait-ce que de façon résumée les chemins théoriques et méthodologiques qui mènent de la linguistique de la parole, des théories de l'énonciation et du dialogisme bakhtinien tel qu'il a été reçu dans les pays de langue romane (dont le Brésil après la France) aux outils de l'analyse de discours que nous avons employés dans cette recherche. Disons rapidement, avec Dominique Maingueneau $(2007, \S 8)$ :

Travailler avec [les genres], c'est à la fois déplacer le regard - du texte vers les dispositifs de communication qu'implique ce texte -, en recourant à une notion foncièrement hybride, inextricablement textuelle et institutionnelle. Si les analystes du discours s'accordent à penser que le genre de discours joue un rôle clé, c'est qu'ils n'appréhendent pas les lieux indépendamment des paroles qu'ils autorisent (contre la réduction sociologique), ni les paroles indépendamment des lieux dont elles sont partie prenante (contre la réduction linguistique).

Nous avons emprunté à la théorie bakhtinienne du langage, telle que l'a développée Bernard Gardin dans ses analyses du discours politique syndicaliste et ouvrier (Gardin, 2005a et 2005b), l'idée que le genre se définit à partir de critères extralinguistiques : les conditions spécifiques et les finalités de l'activité humaine, le contenu thématique, le style, la construction.

Le choix d'un genre se détermine en fonction de la spécificité d'une sphère donnée de l'échange verbal et des besoins de l'objet du sens, de l'ensemble constitué des partenaires, parmi d'autres facteurs. Dans ce contexte, les genres sont relativement stables et les locuteurs les reconnaissent dès les premiers mots. L'énoncé est lié en outre au vouloir-dire et à la relation du locuteur à l'objet dont il parle. Cette relation est marquée par l'expression. Les énoncés portent l'empreinte de leur locuteur, de son jugement de valeur. C'est en fonction de cette valeur que des moyens de la langue sont choisis. Enfin, tout énoncé étant tournévers un allocutaire, il s'oriente selon la réponse présumée de celuici, de ses objections, de ses restrictions. Cette réponse ne peut être constituée qu'en fonction de sa connaissance de la situation, de l'objet du propos, de déterminations politiques, religieuses, morales, etc. Dans un rapport d'influence réciproque, le destinataire est un déterminant de la constitution de l'énoncé et l'énoncé donne une certaine image du destinataire.

On rappellera à ce sujet que la relation entre langage, genre et activité humaine est intrinsèque, sans qu'il soit possible de dresser un répertoire des genres dans la mesure où la liste des activités humaines est illimitée. Certes, la relative stabilité des genres signale le caractère historique, culturellement transmis, de la structure des discours, mais les genres évoluent aussi avec les 
changements sociaux, et leurs variations dépendent des contextes interpersonnels de l'énonciation. Il n'y a pas de formes fixes pour chaque genre; un genre fonctionne comme un schéma sémiotiquement hétérogène, à la fois génétique et génératif, qui s'exprime sous forme de variante.

Ces réflexions permettent de comprendre les formes de la Carta de Princípios, du Manifesto et des Deliberações. Carta de Princípios se traduit simplement en français par "Charte de principes», ce qui donne une clef pour comprendre de quoi il est question : il s'agit d'une déclaration publique. Cela dit, seule une part réduite de son contenu concerne des «principes». Le titre suggère un texte normalisé dont le but serait de fixer des principes d'action, mais le texte lui-même est principalement consacré à l'évaluation de la conjoncture sociale et politique du Brésil à la date de sa rédaction (1979) : les conditions historiques sont réunies pour que naisse au Brésil un parti de gauche radical émanant de la classe ouvrière. Sa phraséologie dogmatique reflète la dynamique d'un groupe d'ouvriers syndicalistes (alphabétisés et instruits dans les cours du soir que leur proposent les mouvements catholiques progressistes) et bien décidés à faire émerger leurs revendications sur le plan politique.

Le Manifesto de 1980 présente un ton beaucoup plus proche du genre "manifeste», avec un ton emphatique et, quant au fond, la proclamation de l'action collective envisagée. Il fait évidemment référence au Manifeste du Parti communiste de Marx et Engels (1848) dont il s'inspire. Émanant d'une organisation, il a un auteur pluriel, marqué par le pronom nós ("nous»). Cependant, l'instance énonciative apparait peu dans le texte, lequel s'énonce généralement à la troisième personne, les faits et les arguments étant sujets des énoncés, ce qui les fait apparaitre comme des vérités indiscutables : d’où un dogmatisme rétrospectivement surprenant.

Les Deliberações de l'Encontro nacional de 2006 sont elles aussi conformes à ce qu'on est en droit d'attendre de «résolutions» d'un «congrès», conclusions d'orientation politique. En l'occurrence, ces Deliberações n'ont pas été soumises au vote comme des motions opposées à d'autres motions : elles résultent d'un consensus empirique, plateforme de la campagne électorale de la présidentielle qui va s'achever, quelques mois plus tard, par la réélection de Lula.

\section{De la Carta au Manifesto}

Le Manifesto interagit avec les voix qui ont suscité la Carta; il leur répond, les accueille ou bien les nie en les ignorant. Sans détailler ici les correspondances entre les deux textes, nous présenterons certaines des différences qui permettent de suivre l'évolution des textes organiques du Parti des Travailleurs dans l'histoire de ce parti. 
L'analyse de la Carta et du Manifesto, textes dont le premier est de dix mois antérieur au second, permet de repérer ce que nous appelons des condensations, opérées par effacement, réduction et déplacement d'accent, procédés par lesquels les thèmes du premier texte sont repris et modifiés par le second, ainsi que des ajouts ${ }^{6}$. La condensation procède d'abord par omission et réduction - certains contenus de la Carta n'apparaissent plus dans le Manifesto et d'autres y sont réduits -, puis par déplacements d'accent - les rédacteurs du Manifesto prêtent leurvoix aux idées reprises dans la Carta mais modifient leur signification et leur orientation globale ; les fondateurs du parti, notamment, n’apparaissent plus comme des ouvriers de la métallurgie. Quant aux ajouts, figure inverse de la condensation, ce sont de nouveaux contenus, des développements d'idées.

La Carta commence parvoir une nécessité historique dans la formation d'un parti propre aux travailleurs en affirmant que l’idée en est « aussi vieille que la classe ouvrière elle-même », affirmation que contrarient en partie les prétentions de nouveauté et l'idée d'un murissement progressif des travailleurs brésiliens que le Manifesto présente comme le produit d'un déterminisme historique :

Il a commencé à devenir de plus en plus clair pour les mouvements populaires que leurs luttes immédiates et spécifiques ne sont pas suffisantes pour garantir la conquête des droits et des intérêts du peuple travailleur. C'est pourquoi est apparue la proposition de Parti des Travailleurs. ${ }^{7}$

Les deux textes partent de l'objectif de réaliser une société égalitaire. Cet énoncé du premier : «Le Parti des Travailleurs se définit, programmatiquement, comme un parti qui a pour objectif d'en finir avec la relation d'exploitation de l'homme par l'homme ${ }^{8}$, est ainsi reformulé par le second : «Le Parti des Travailleurs cherchera à conquérir la liberté pour que le peuple puisse construire une société égalitaire, où il n'y ait ni exploités ni exploiteurs. »9

Les deux textes renvoient aux mêmes champs lexicaux que le Manifeste du Parti communiste de Marx et Engels : on y retrouve la même affirmation de la lutte des classes (« [Notre société] est basée sur l'exploitation et l'inégalité entre les classes ${{ }^{10}}^{10}$ ), la même métaphore guerrière de la confrontation violente entre exploiteurs et exploités, oppresseurs et opprimés. On y retrouve le

6. Ces catégories d'analyse sont empruntées à Cunha (1992).

7. «Começou a tornar-se cada vez mais claro para os movimentos populares que as suas lutas imediatas e específicas não bastam para garantir a conquista dos direitos e dos interesses do povo trabalhador. Por isso, surgiu a proposta do Partido dos Trabalhadores. » (Manifesto)

8. "O Partido dos Trabalhadores define-se, programaticamente, como um partido que tem como objetivo acabar com a relação de exploração do homem pelo homem. » (Carta)

9. "O Partido dos Trabalhadores buscará conquistar a liberdade para que o povo possa construir uma sociedade igualitária, onde não haja explorados nem exploradores. » (Manifesto)

10. "[A nossa sociedade é] baseada na exploração e na desigualdade entre as classes.» (Carta) 
même antagonisme entre, d'un côté, les exploiteurs, détenteurs de privilèges et de pouvoir, c'est-à-dire les classes dominantes, propriétaires du capital, qui contrôlent l'appareil d'État et ont soif de privilèges, et de l'autre les exploités, sans privilèges et sans pouvoir, «le peuple brésilien [qui] est pauvre, en mauvaise santé et n'est jamais parvenu à prendre part aux décisions qui engagent le destin du pays ${ }^{11}$, parce que sont ignorés les intérêts des masses exploitées, depuis toujours marginalisées économiquement et politiquement :

Le gouvernement déclenche la répression [...]. Les partis et les gouvernements créés et dirigés par les patrons et par les élites politiques [...]. Les patrons et le gouvernement ont eu besoin de se donner la main [...]. Les patrons emploient tous les moyens à leur portée pour casser l'unité des travailleurs [...]. ${ }^{12}$

\section{Enfin, la Carta exclut longuement toute «alliance de classes» :}

Le Parti des Travailleurs se refuse à accepter en son sein des représentants des classes exploiteuses. Autrement dit, le Parti des Travailleurs est un parti sans patrons! [...] La masse travailleuse [est] l'unique et vrai sujet et agent d'une démocratie effective [...]. Le Parti des Travailleurs pose que l'émancipation des travailleurs est l'œuvre des travailleurs eux-mêmes [...].Un parti uniquement de travailleurs [...]. Les exploités et les opprimés ont le besoin permanent de s'organiser séparément [...]. Le Parti des Travailleurs se déclare ouvert à la participation de toutes les couches salariées du pays [...]. S’emparer du pouvoir politique et implanter le gouvernement des travailleurs, basé sur les organes de représentation créés par les masses travailleuses elles-mêmes en vue d'une démocratie directe primordiale [...]. ${ }^{13}$

Contrastivement, le Manifesto fait part à la société brésilienne tout entière de la création du parti comme du résultat des mobilisations politiques organisées par un éventail très ouvert de secteurs professionnels et de groupes sociaux. Ce qui est formulé dans des expressions pluralistes et, jusqu'à un certain point, contradictoires. La théologie de la libération, les positions progressistes de l’Église catholique, ses formations syndicales et ses mouvements

11. «O povo brasileiro está pobre, doente e nunca chegou a ter acesso às decisões sobre os rumos do país. " (Carta)

12. «O governo desencadeia sua repressão [...]. Partidos e governos criados e dirigidos pelos patrões e pelas elites políticas [...]. Os patrões e o governo precisaram dar-se as mãos [...]. Os patrões usam de todos os meios a seu alcance para quebrar a unidade dos trabalhadores. " (Carta)

13. "O Partido dos Trabalhadores recusa-se a aceitar em seu interior, representantes das classes exploradoras. Vale dizer, o Partido dos Trabalhadores é um partido sem patrões! [...] A massa trabalhadora, único e verdadeiro sujeito e agente de uma democracia efetiva [...]. O Partido dos Trabalhadores entende que a emancipação dos trabalhadores é obra dos próprios trabalhadores [...]. Um partido só dos trabalhadores [...]. Os explorados e oprimidos têm permanente necessidade de se manter organizados à parte [...]. O Partido dos Trabalhadores se declara aberto à participação de todas as camadas assalariadas do país [...]. Os patrões usam de todos os meios a seu alcance para quebrar a unidade dos trabalhadores [...]. Apoderar-se do poder político e implantar o governo dos trabalhadores, baseado nos órgãos de representação criados pelas próprias massas trabalhadoras com vista a uma primordial democracia direta [...]. » (Carta). On aura reconnu ce que les Russes, en 1917, avaient nommé des soviets. 
d'éducation populaire s'opposent au marxisme qui triomphe à Cuba et aux résurgences du populisme d'avant 1964, d'une part, tandis que les origines bourgeoises et petites-bourgeoises de la classe intellectuelle brésilienne ralliée au parti contribuent elles aussi aux tensions. Toujours est-il que la synthèse s'est faite sur la volonté commune de précipiter le départ des généraux et d'accéder à une démocratie permettant de transformer le Brésil en une société égalitaire. Le Parti des Travailleurs se présente ainsi comme le moyen de lancer dans l'action politique les ouvriers de l'industrie et le prolétariat rural, très majoritaires dans le pays :

Nous sommes un Parti des Travailleurs, pas un parti pour tromper les travailleurs [...]. Un parti ample et ouvert à tous ceux qui s'engagent pour les causes des travailleurs [...]. [Le Parti] affirme son engagement en faveur d'une démocratie pleine et entière, exercée directement par les masses [...]. Il luttera [...] pour la démocratisation de la société à tous les niveaux [...]. [Il proclame] la solidarité avec la lutte de toutes les masses opprimées dans le monde. ${ }^{14}$

On voit que le vocabulaire du Manifesto continue de refléter l'opposition « dominant», « exploiteur», «privilégié » contre «dominé», «exploité», «opprimé». Il reproduit les oppositions dualistes propres au marxisme, du type «masses vs "élites», "opprimés» vs "oppresseurs», «classe ouvrière » vs «patrons», «travailleurs» vs «élites», etc. D’un autre côté, il atténue les expressions de caractère ouvriériste; n’y figurent plus les limites précédemment fixées à de possibles alliances de classe, ni les références au gouvernement des généraux, que ce soit en relation avec la répression ou en tant qu'allié objectif des patrons et des élites. Le Manifesto déplace aussi les lignes en reformulant l'appel au socialisme, la clausule de la Carta - «car il n'y a pas de socialisme sans démocratie ni de démocratie sans socialisme» - étant retranchée :

Le Parti des Travailleurs affirme son engagement en faveur d'une démocratie pleine, exercée directement par les masses, car il n'y a pas de socialisme sans démocratie ni de démocratie sans socialisme. ${ }^{15}$

Le Parti des Travailleurs affirme son engagement en faveur d'une démocratie pleine exercée directement par les masses. ${ }^{16}$

D’un autre côté, le Manifesto présente des ajouts que l'on peut résumer ainsi :

14. "Somos um Partido dos Trabalhadores, não um partido para iludir os trabalhadores [...]. Um partido amplo e aberto a todos aqueles comprometidos com as causas dos trabalhadores [...]. Afirma seu compromisso com a democracia plena e exercida diretamente pelas massas [...]. Lutará [...] pela democratização da sociedade em todos os níveis [...]. [Proclama a] solidariedade à luta de todas as massas oprimidas do mundo. " (Manifesto)

15. "O Partido dos Trabalhadores afirma seu compromisso com a democracia plena, exercida diretamente pelas massas, pois não há socialismo sem democracia nem democracia sem socialismo.» (Carta)

16. "O Partido dos Trabalhadores afirma seu compromisso com a democracia plena e exercida diretamente pelas massas. » (Manifesto) 
pour dépasser une situation dans laquelle la grande majorité des Brésiliens sont devenus des citoyens de deuxième classe, il est possible d'organiser la participation des travailleurs à la construction d'une démocratie ${ }^{17}$. C'est ainsi que le Manifesto vient contredire la Carta sur trois points essentiels.

En premier lieu, il élargit l'éventail des intéressés au monde rural, à l'ensemble du prolétariat suburbain et du sous-prolétariat, d'un côté, aux classes moyennes de l'autre, et il réduit l'importance de l'autonomie organisationnelle du Parti des Travailleurs, désormais considéré comme un simple «outil de construction d'une société qui réponde aux intérêts des travailleurs et des autres secteurs exploités par le capitalisme» (nous soulignons) ${ }^{18}$.

Il fait ensuite le lien entre l'accession à la démocratie et une démarche d'éducation et d'encadrement des catégories sociales les plus défavorisées, jusqu'alors abandonnées à elles-mêmes et consécutivement indifférentes à la politique :

[La démocratie viendra] par les organisations de base de la société, [...] par la construction d'une structure interne démocratique, [...] par la démocratisation de la société à tous les niveaux, [...] par des syndicats et des partis indépendants de l'État [...]. Les décisions économiques doivent être soumises aux intérêts populaires [à travers des représentations] fondées sur les organisations de base..$^{19}$

Enfin, dans le même esprit, à l'affirmation que le Brésil « ne sera vraiment indépendant que lorsque l'État sera dirigé par les masses travailleuses», le Manifesto ajoute qu'il est nécessaire que «l'État devienne l'expression de la société $»^{20}$. La corrélation entre la vocation des masses à exercer le pouvoir et l'expression de la volonté de la société est placée dans une perspective historique à long terme.

En résumé, le Manifesto ne polémique pas avec la Carta, il ne la cite pas et ne lui répond pas explicitement, pas plus qu'il ne définit ses auteurs, à la différence de la Carta dont les signataires se firent une obligation de se définir en termes de classe: « Nous, dirigeants syndicaux, ne prétendons pas être les propriétaires du Parti des Travailleurs [...]. Nous avons conscience que dans ce rôle, en ce moment, nous sommes irremplaçables, et ce n'est qu'en considération de cela que nous revendiquons le rôle de créateurs du Parti des Travailleurs. ${ }^{21}$

17. Cette inflexion se fait encore sentir dans le discours du Parti et rattache le Manifesto aux Declarações.

18. "[O Partido dos Trabalhadores é uma mera] ferramenta da construção de uma sociedade que responda aos interesses dos trabalhadores e dos demais setores explorados pelo capitalismo. » (Ibid.)

19. "[...] organizações de base da sociedade [...] [por] construir uma estrutura interna democrática, [...] [pela] democratização a sociedade em todos os níveis, [...] [por] sindicatos e partidos independentes do Estado [...]. As decisões sobre a economia se submetam aos interesses populares [através de representações] fundadas nas organizações de base. » (Ibid.)

20. "[O Brasil] só será verdadeiramente independente quando o Estado for dirigido pelas massas trabalhadoras [...]. O Estado se torne expressão da sociedade. » (Ibid.)

21. «Nós, dirigentes sindicais, não pretendemos ser donos do Partido dos Trabalhadores [...]. Temos 
Le dialogue du Manifesto avec la Carta ressemble ainsi à ce que Bakhtine appelle une polémique secrète :

[...] le mot de l'autre reste en dehors du discours de l'auteur, mais le discours de l'auteur en tient compte et lui est rapporté. Ici le mot de l'autre, au lieu d'être reproduit avec un sens nouveau, exerce son action, son influence et, d'une façon ou d'une autre, détermine le mot de l'auteur, tout en demeurant lui-même hors de celui-ci. C'est la situation du mot dans la polémique secrète et, en général, dans la réplique d'un dialogue. (Bakhtine, 1970, p. 227)

Si le Manifesto ne fait référence à aucun document antérieur - pas même à la Carta -, le discours d'identité qui lui est propre se retrouve, au sein de ses statuts actuels, dans des mots qui peuvent paraitre comme la synthèse officielle de l'idéal du parti - l'engagement dans des luttes militantes en faveur du socialisme démocratique :

Art. 1 : Le Parti des Travailleurs (PT) est une association volontaire de citoyennes et de citoyens qui se proposent de lutter pour la démocratie, le pluralisme, la solidarité, les transformations politiques, sociales, institutionnelles, économiques, juridiques et culturelles, destinées à éliminer l'exploitation, la domination, l'oppression, l'inégalité, l'injustice et la misère, dans le but de construire le socialisme démocratique. ${ }^{22}$

Les effacements révèlent la relation tendue entre les voix, courants et tendances idéologiques qui traversaient le discours du Parti des Travailleurs avant même qu'il eût commencé d'exister.

\section{Les Deliberações}

Près de trente ans après la naissance du parti et plus de trois ans après l'accession au pouvoir, on observe dans les Deliberações de nombreux objets discursifs nouveaux : les thèses centrales traitent du combat contre le néolibéralisme et de l'importance de la réélection de Lula pour l'avenir du parti. Sont élaborées les bases politiques de la campagne présidentielle. Sont abordés les «scandales» (trafic d'influence, concussion, prévarication..., le lot ordinaire de la vie politique brésilienne) impliquant certains responsables du parti. On discute du problème du «cout de la démocratie» dans une société capitaliste et des mesures destinées à en diminuer l'impact, une thématique dans laquelle résonnent avec force les accusations lancées par les grands médias libéraux contre le Parti des Travailleurs, accusé d'acheter les voix de députés d'opposition

a consciência de que, nesse papel, neste momento, somos insubstituíveis, e somente em vista disso é que nós reivindicamos o papel de lançadores do Partido dos Trabalhadores. » (Carta)

22. "Art. 1ํ: O Partido dos Trabalhadores (PT) é uma associação voluntária de cidadãs e cidadãos que se propõem a lutar por democracia, pluralidade, solidariedade, transformações políticas, sociais, institucionais, econômicas, jurídicas e culturais, destinadas a eliminar a exploração, a dominação, a opressão, a desigualdade, a injustiça e a miséria, com o objetivo de construir o socialismo democrático. » (Ibid.) 
corrompus. Sont développées des réflexions sur la transition d'un modèle de société à un autre. Est souligné le poids des forces conservatrices qui implique des alliances électorales. Le fait que le Parti des Travailleurs soit aux affaires implique d'assumer certaines contradictions. On souligne l'importance de renforcer la démocratie représentative et participative; on définit le projet de gouvernement comme d'inspiration démocratique et populaire, avec des restrictions sur certaines options de politique économique.

Face à la théorie néolibérale du «moins d'État», le texte défend un État fort au service de tous comme transition vers un socialisme qui reste à construire dans la vie quotidienne. En accord avec cette conception, les Deliberações traitent du thème de l'exclusion d'une façon qui renvoie aux aspirations du Manifesto en pointant le fait que ce sont les gouvernements démocratiques et populaires locaux et fédéraux qui ont non seulement changé en mieux les politiques sociales, mais aussi mis en pratique des formes innovantes de participation populaire (l'exemple de Porto Alegre a été rapporté dans le monde entier). Le Programme de gouvernement exprimé par la Treizième rencontre nationale du Parti des Travailleurs n'oublie pas que ce dernier se destine à diriger un État qui a contracté depuis plusieurs siècles une gigantesque dette sociale auprès de la majorité de la population, reléguée dans une situation perverse d'exclusion sociale ou, dans les termes du Manifesto, d'« exploitation ».

Les Deliberações traitent longuement de la «conjoncture internationale». Les items introductifs insistent fortement sur «les avancées de la gauche politique et sociale en Amérique latine et de l'intégration latino-américaine », ainsi que sur l'hégémonie des forces néolibérales qui soutiennent l'ordre international en vigueur - tout en la remettant fortement en question étant donné que «les riches n'ont aucun projet d'avenir à proposer aux pauvres dans le monde ${ }^{23}$. Le Parti des Travailleurs doit avoir en conséquence « une claire implication internationale » pour "développer son action en Amérique Latine, sans que cela gêne [sa] présence active en Afrique, en Europe et en Asie», notamment «auprès de ce que l'on nomme le mouvement altermondialiste tel qu'il s'exprime principalement dans le Forum social mondial»; il doit mettre en place «un plan d'intégration économique, politique et culturelle» et investir «dans le renforcement du Mercosul et de la Communauté sud-américaine des Nations ${ }^{24}$. Tout un programme qui, à la fois, met en avant et produit des stratégies politiques compatibles avec l'esprit du Manifesto, où cependant elles ne se trouvent pas explicitement formulées :

23. "Os ricos não têm um projeto de futuro a oferecer aos pobres do mundo. " (Deliberações)

24. "O Partido dos Trabalhadores deve ampliar sua atuação na América Latina, sem que isso impeça nossa presença ativa na África, Europa e Ásia [...] uma participação ativa junto ao chamado movimento altermundista, expresso principalmente no Fórum Social Mundial [...] um plano de integração econômica, política e cultural [...] [investir] no fortalecimento do Mercosul e da Comunidade sul-americana das Nações. " (Ibid.) 
L'opposition de droite et ses alliés internationaux ont conscience de l'importance stratégique de l'élection présidentielle brésilienne. Les forces néolibérales veulent récupérer le contrôle du gouvernement fédéral, reprendre la répression des mouvements sociaux, la soumission aux intérêts nord-américains, l'idéologie et la pratique de l’État minimum et des privatisations. ${ }^{25}$

La présence de Lula à la présidence du Brésil constitue un point d'appui pour que se crée, dans la société brésilienne, une culture politique qui l'emporte sur l'hégémonie des élites sur la politique du Brésil. ${ }^{26}$

Dans les Deliberações, une bonne part du lexique du Manifesto perd de son importance. C'est ainsi que le mot trabalhador est 70 fois moins employé en 2006 qu'en 1980. Cette évolution est le signe d'un affaiblissement de la position des travailleurs eux-mêmes dans l'élaboration du texte, dans la mesure où ce mot relève moins ici de la tradition marxiste qu'il ne qualifiait originellement l'énonciateur. L'ancrage du Parti des Travailleurs dans les milieux ouvriers et sa nature «classiste» sont indiscutablement affaiblis par le passage de l'utopie de l'émancipation des travailleurs, caractéristique du Manifesto, à une concrétisation transitoire et limitée de cette utopie, la construction d'un pays postnéolibéral par le gouvernement Lula.

Avec le mot socialisme, absent du Manifesto, les Deliberações reprennent une formulation de la Septième rencontre nationale (1990) qui programme la future relation entre Parti des Travailleurs et société socialiste : «Le socialisme pétiste [du Parti des Travailleurs] - notre horizon stratégique - est une construction historique et non un objectif abstrait à atteindre. ${ }^{27}$

Le texte de 2006 dialogue ainsi avec des positions qui, dans le Parti ou hors de lui, défendent le socialisme comme un modèle et voient dans son avènement une nécessité historique.

La dialectique «privilège » vs « exploitation », caractéristique du Manifesto, est remplacée lors de la Treizième rencontre par l'opposition «exclusion »vs «insertion sociale». C'est en ce sens que des politiques compensatoires de type RMI sont considérées comme des mesures importantes.

Pour mieux illustrer, à partir d'une approche quantitative, la spécificité de chacun de nos trois textes, le tableau 1 ci-dessous permet de comparer quelques données lexicométriques ${ }^{28}$ :

25. "A oposição de direita e seus aliados internacionais têm consciência da importância estratégica da eleição presidencial brasileira. As forças neoliberais querem recuperar o controle do governo federal, retomando a repressão contra os movimentos sociais, a submissão aos interesses norteamericanos, a ideologia e a prática do Estado mínimo e das privatizações. » (Ibid.)

26. "A presença de Lula na presidência do Brasil constitui um ponto de apoio para que se crie, na sociedade brasileira, uma cultura política que supere a hegemonia das elites na política brasileira.»

27. "O socialismo petista-nosso horizonte estratégico - é uma construção histórica e não um objetivo abstrato a ser atingido. "

28. Nous ne pouvons aborder que par allusion la méthode d'analyse retenue; ce tableau 1 n'est donné que comme un échantillon des résultats bruts obtenus par décompte d'occurrences de mots clés du discours politique brésilien. 


\begin{tabular}{|c|c|c|c|}
\hline & Carta & Manifesto & Deliberações \\
\hline Taille des textes* * & 2,5 & 1 & 14,4 \\
\hline $\begin{array}{l}\text { Partido dos Trabalhadores / PT / nosso partido / } \\
\text { Partido / partido }\end{array}$ & 38 & 15 & 104 \\
\hline $\begin{array}{l}\text { Governo Lula / gestão Lula / o - deste - nosso } \\
\text { - governo / Governo Federal / presidência } \\
\text { da República / o segundo mandato }\end{array}$ & & & 134 \\
\hline $\begin{array}{l}\text { classe(s) / dominantes / explorada(s) / } \\
\text { produtoras / trabalhadora(s) }\end{array}$ & 11 & - & 2 \\
\hline $\begin{array}{l}\text { trabalhador(es) / trabalhadores produtores / } \\
\text { população trabalhadora / povo trabalhador }\end{array}$ & 36 & 21 & 11 \\
\hline $\begin{array}{l}\text { massas trabalhadoras / populares / oprimidas / } \\
\text { exploradas / marginalizadas }\end{array}$ & 21 & 8 & 0 \\
\hline $\begin{array}{l}\text { Explorados / superexploração / } \\
\text { setores explorados / excluído(a)(s) / minorias }\end{array}$ & 7 & 8 & 5 \\
\hline $\begin{array}{l}\text { Lula / o Presidente / o Presidente Lula / } \\
\text { o presidente da República }\end{array}$ & - & - & 21 \\
\hline $\begin{array}{l}\text { democracia / democrático / democratização / } \\
\text { forças democráticas e populares }\end{array}$ & 31 & 7 & 72 \\
\hline $\begin{array}{l}\text { luta(s) / lutar / lutará / nossa luta / } \\
\text { lutas sociais / lutas populares }\end{array}$ & 15 & 11 & 7 \\
\hline greve / grevista / direito de greve / movimento & 15 & 2 & 2 \\
\hline $\begin{array}{l}\text { reeleição / reeleger Lula / candidatura / } \\
\text { campanha Lula / eleições / eleição presidencial / } \\
\text { Programa de Governo / aliados / alianças / } \\
\text { coligações / coalizão / aliados internacionais }\end{array}$ & 2 & - & 61 \\
\hline $\begin{array}{l}\text { corrupção / corruptora(s) / patrimônio ético / } \\
\text { ética }\end{array}$ & - & - & 17 \\
\hline socialismo / sociedade socialista & 3 & - & 5 \\
\hline neoliberal(is) / neoliberalismo / liberalismo & - & - & 30 \\
\hline
\end{tabular}

* En gras, les fréquences les plus significatives, y compris du fait de la rareté ou de l'absence.

* * La taille du Manifesto a servi d'unité de comparaison avec les deux autres.

Tableau 1. Comparaison des fréquences de mots dans les trois textes

Une constatation émerge des références aux syndicats et au gouvernement. Le mot governo lui-même est pratiquement absent du Manifesto et de la Carta mais revient souvent dans les Deliberações : sans aucun doute, c'est un indice de la grande préoccupation des rédacteurs pour l'exercice du pouvoir et le maintien aux affaires, tandis que faute de combats épiques - à la différence du passé -, la référence aux syndicats tend à disparaitre.

On constate encore que le Manifesto a éliminé le mot classe(s) et réduit les occurrences de greve(s) et de luta(s) tandis que massa(s) résiste. Dans 
les Deliberações, massa(s) disparait à son tour tandis que la fréquence de trabalhador(es), trabalhadora(s), adjectif et nom, diminue fortement. On trouve à leur place, pour désigner les sujets de l'Histoire, des expressions évoquant le Brésil et la société (brésilienne). De même, explorado $(a / s)$ est remplacé par exclu(e/s), adjectif et nom. Un vocabulaire caritatif emprunté aux ONG et à la gauche chrétienne remplace le vocabulaire politique d'origine marxiste encore en vigueur dans les années quatre-vingt et quatre-vingt-dix.

Une saillance commune aux trois discours est la récurrence sensible de democratia, mais on relève en particulier dans les Deliberações l'apparition de thèmes tels que le "néolibéralisme», la «corruption des politiques», le problème des «alliances» entre partis et la mise en avant des questions électorales : candidatura, reeleição, programa de governo, etc. : ce discours s'inscrit dans l'actualité, ce qui s'explique en bonne partie par son genre (actes d'un congrès), nettement différent de la nature programmatique et instituante de la Carta et du Manifesto.

Leur comparaison permet de relever des convergences et des divergences entre les textes fondateurs et la plate-forme de 2006 . Permanences et effacements répondent à de nouvelles contraintes historiques et idéologiques, politiques et sociales. Le Parti des Travailleurs est resté fidèle à ses orientations d'origine mais, en modernisant ses thématiques et ses formulations pour élargir sa base électorale, il a gommé l'accent mis au départ sur la lutte des classes et la rhétorique révolutionnaire.

L'analyse des Deliberações montre qu'actuellement, pour les sujets du discours, être au pouvoir, avoir le pouvoir, appartenir au Parti des Travailleurs compte plus que telle ou telle idéologie : l'exercice du pouvoir semble importer davantage que la construction d'une société nouvelle, même reformulée en lutte contre les exclusions, sans qu'il soit possible de dire si cet assagissement ou cette normalisation, cette stabilisation ou cette institutionnalisation de l'action politique des gauches brésiliennes fédérées autour du président de la République est un gage de pérennité puis, à plus ou moins long terme, de réalisation concrète de ses aspirations initiales. À cette question problématique, il ne semble pas que l'analyse de discours ait vocation à répondre. Ce que nous retiendrons en revanche, c'est que cette évolution représente une mutation discursive de grande ampleur dont l'existence même n'était pas avérée au départ, la mise en mots du politique étant devenue progressivement, au cours de ces trente dernières années, une affaire de professionnels de la communication, et l'expression de voix totalement séparées de la classe ouvrière et du sous-prolétariat urbain et rural modernes qu'elles sont censées représenter ${ }^{29}$.

29. Voir Bourdieu, Thompson, 2001. 


\section{Références}

Alves Maria Helena Moreira, 2005, Estado e oposição no Brasil (1964-1984), São Paulo, Edusc.

AuthIER-REVUz Jacqueline, 1982, "Hétérogénéité montrée, hétérogénéité constitutive. Éléments pour une approche de l'autre dans le discours, Documentation et recherche en linguistique allemande contemporaine, $\mathrm{n}^{0}$ 26, p. 91-151.

Bakntine Mikhaïl, 1970, Problèmes de la poétique de Dostoïevski, Lausanne, L’Âge d'Homme.

- 1984, Esthétique de la création verbale, Paris, Gallimard (2000, Estética da criação verbal, São Paulo, Martins Fontes).

- 2003, Pour une philosophie de l'acte, Lausanne, L’Âge d'Homme.

BAKHTIne Mikhaïl (Volochinov Valentin N.), 1977, Le marxisme et la philosophie du langage. Essai d'application de la méthode sociologique en linguistique, Paris, Minuit.

Bourdieu Pierre, Thompson John B., 2001, Langage et pouvoir symbolique, Paris, Le Seuil (édition augmentée de Ce que parlerveut dire, Paris, Fayard, 1982).

CunHA Doris, 1992, Discours rapporté et circulation de la parole, Louvain-la-Neuve, Peeters.

FERREIRA Jorge, 2003, O governo Goulart e o golpe civil-militar de 1964, Rio de Janeiro, Editora Civilização Brasileira.

FRANçoIs Frédéric, 2006, "Dialogisme des "voix" et hétérogénéité constitutive du "sens". Le "savoir", le "quotidien” et le "littéraire”, communauté et différences d'accentuation chez Voloshinov, Bakhtine et Vygotzki. Une contribution indirecte à la pédagogie du "texte littéraire” ", Investigações. Linguística e teoria literária, vol. XIX, $\mathrm{n}^{\circ} 2$.

GARDIN Bernard, 2005a, Langage et luttes sociales, N. Gardin, F. François éd., Limoges, Lambert-Lucas.

- 2005b, Paroles d'ouvrières et d'ouvriers, N. Gardin, J. Boutet éd., Limoges, Lambert-Lucas.

Maingueneau Dominique, 2005a, Genèse du discours, Paris, Mardaga (Gênese dos discursos, Curitiba, Criar).

- 2005b, Paratopie et scène d'énonciation, Paris, Armand Colin (Cenas da Enunciação, Curitiba, Criar).

- 2007, "L'idéologie. Une notion bien embarrassante», Contextes, $n^{0} 2$, février, en ligne [URL: http://contextes.revues.org/index189.html]. Consulté le 19 avril 2010.

MoIRAND Sophie, 1999, "Les dimensions dialogiques d'une catégorie discursive : l'explication », Jalons pour le $75^{e}$ anniversaire de l'enseignement du français à l'Université de Turku, Université de Turku (Finlande), p. 71-87.

- 2005, «Le dialogisme entre problématiques énonciatives et théories discursives », Cahiers de praxématique, $\mathrm{n}^{\circ}$ 43, p. 189-220.

- 2006a, «Entre discours et mémoire. Le dialogisme à l'épreuve de la presse quotidienne», Tranel (Travaux neuchâtelois de linguistique), $\mathrm{n}^{\circ} 44$, Intertextualité et interdiscours dans les médias, Neuchâtel, Université de Neuchâtel. 
- 2006b, «Responsabilité et énonciation dans la presse quotidienne. Questionnements sur les observables et les catégories d'analyse», Semen, $\mathrm{n}^{0} 22$, en ligne [URL: http://semen.revues.org/document2798.html]. Consulté le 20 avril 2010.

SAntAnA Marco A., 2003, "Trabalhadores em movimento", O Brasil Republicano, vol. IV, O tempo da ditadura, L. de A. N. Delgado, J. Ferreira éd., Rio de Janeiro, Civilização Brasileira.

SkIDMORE Thomas, 1988, Brasil. De Castelo a Tancredo, São Paulo, Paz e Terra.

\section{Sources}

PARTIDO dos TRABALhADORES, archives en ligne [URL : http://www.pt.org.br/portalpt/ documentos/documentos-historicos-32/pagina-1/]. Consulté le 20 avril 2010.

- 2002, Trajetórias, São Paulo, Editora Fundação Perseu Abramo.

- 2005, Resoluções de encontros e congressos e programas de governo: 1979-2002, São Paulo, Bartira. 